\title{
Quantitative Training of American Football Kickers Using Real-Time Sensing and Prediction
}

\author{
Aaron Stout, Ehren Biglari, Jacob Kantor, Cole Meyers,Alyssa Schaefbauer, Yusheng Feng* \\ Center for Simulation, Visualization and Real-Time Prediction (SiViRT) \\ The University of Texas at San Antonio \\ San Antonio, Texas 78249, U.S.A. \\ *e-mail: yusheng.feng@utsa.edu \\ website: http://sivirt.utsa.edu
}

\begin{abstract}
American football plays a large part in the American culture. During the season, football fans bleed the colors of their favorite team and are extremely enthusiastic about every game. Kicking is a very fundamental and vital part of the game. This paper presents a training system that integrates wireless technology, computer simulation, and advanced visualization. This system is capable of providing quantitative training to kickers and allows coaches to design effective and unique training programs. Moreover, the data collected in real-time can be used to calibrate the footballs dynamic model in predicting the final position with respect to the field goal, which is then visualized using 3D stereoscopic technology.
\end{abstract}

$\begin{array}{llcc}\text { Keywords-American } & \text { Football; } & \text { Kicker } & \text { Training; } \\ \text { mathematical Modeling; } & \text { Wireless } & \text { Sensing; } & \text { Trajectory } \\ \text { Prediction } & & & \end{array}$

\section{INTRODUCTION}

In American football, a kicker is heavily relied on for scoring points after every touch down and for field goals. It is often crucial to either win or loss of a game, which is determined by a margin of a few points. More often than not, one kick at the closing minutes can be the determining factor. There are training camps across the Unites States that specialize in football kicking. Some of these camps have a whole team of coaches helping one kicker to improve. Although these camps are helpful, they are usually observation-based and not quantitative. A well-trained kicker is set himself apart from the rest by his ability to be consistent and reliable. While a training camp is helpful, it is hard to maintain the consistency without continuous reinforcement. Moreover, a kicker on the team lacks specialized practicing when he is trained with the rest of the team since the focus is on offensive and defensive approaches and drills. The objective of this football-kicking simulator and training system is to meet the need of a kicker and provide a real-time training tool that would give a kicker the ability to practice off the field and receive quantitative feedback for player-specific training via real-time flight data collection and computer modeling for their leg motion, foot-and-ball contact as well as trajectory prediction.Moreover, the data collected in real-time can beused to calibrate the football dynamic model inpredicting thefinalposition with respect to the field goal, which is then visualized using 3D stereoscopic technology.

\section{Overall System DesigN}

\section{A. Design Goal}

The simulation and training system is designed to be an indoor environment that allows a kicker to practice under realistic game situations by computer simulation and visualization. The system consists of major components of 1) wall-mounting pressure sensing device, 2) wireless data collection device embedded in the football, 3) data analysis and model prediction system, and 4) visualization system that emulates football stadiums with predicted football trajectory. In addition, the system incorporates what a training camp can offer with coach assistance. The advantage of the system is to provide a practice environment where a kicker can practice no matter the time of day or weather conditions outside. Moreover, this training system incorporates a quantitative feedback of the trajectory of the football for players to determine if their kick would be within the field goal. A pressure-sensing matcan be adopted for a visual representation of the kicker's footprint trail in order to inspect for consistency; and the high-speed cameras for replay and evaluation of body movements of how the kicker follows through after kicking. To create an environment as realistic as possible, the kicker not only can visualize theresult of kicking to see if the ball reaches the field goal on the screen, but also have the option of hearing the sound of rush of a crowed and the pressure of a clock. This design will help kickers as well as their coaches, to enable them to design specific game strategyfor better performance.

\section{B. System Design}

1) Mechanical Framework and Design Analysis. The system frame, shown in Figure 1, of the Football Kicking Simulator (FKS) is designed to be easily mounted on a wall similar to most current television sets. The mounting system consists of two main components: the sensor panel apparatus that houses the sensors on the front andcrossbeam supports on the rear. The wall mount portion comprises a separate part braced by two vertical beams 
and three horizontal beams. The wall mount support spacing is based upon the industrial standards for stud separation in both residential and commercial buildings that require 16" and 24" separation between studs. Mounting directly to the wall allows greater room for the rest of the simulation and for optimal stability.
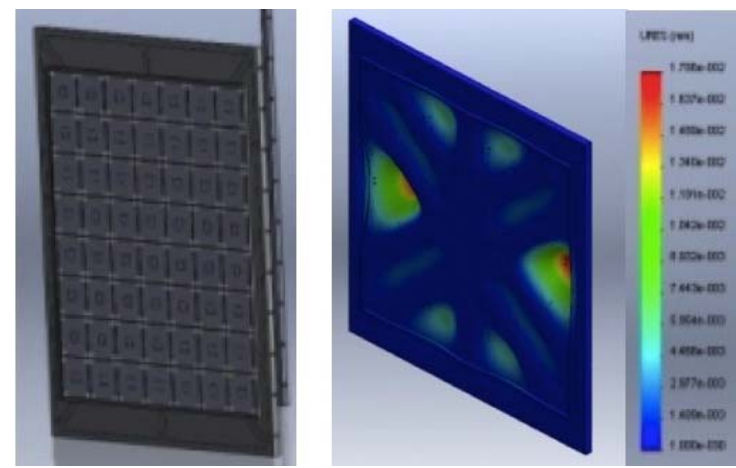

FIGURE 1 FRAME ASSEMBLY AND ITS STRESS ANALYSIS UNDER THE WORST-CASE SCENARIO IMAPCT OF FOOTBALl KICKING. THE Frame SUPPORT IS DESGINED AND OPTIMIZED TO SUSTAIN THE MAXIMUM STRESS

The design was drafted in SolidWorks ${ }^{\circledR}$ allowing material constraints to be optimized using SolidWorks ${ }^{\circledR}$ Design Study Module.The sensor panel support with crossbeams is to increase the strength of overall system. Since this component is the large and heavy, we conducted shape and size optimization using the built-in design study. Utilizing a multi-objective optimization algorithm in SolidWorks ${ }^{\circledR}$, the design parameters such as wall thickness and crossbeam length are optimized to reduce weight and provide optimum support. A basic force simulation verifying structure support is shown in Fig. 1 from the first phase of development. With a force of $200 \mathrm{~N}$ applied uniformly to the surface of the frame, assuming the material is 1060 alloy steel and the rear side of the frame is stationary, the maximum deflection is limited to $0.018 \mathrm{~mm}$ and the minimum factor of safety is $46.94 \mathrm{~mm}$, which is well above the required minimum.

\section{Pressure SENSORS AND TheIr MOUNTING}

To calculate trajectory of the football, three positioning functions with respect to time are needed. For the purpose of model calibration, we measure the time interval between the moment when the ball leaves the ground and that when it hits the screen as well as the angle and distance that the ball travels from the floor to the screen. Force sensors are being used to determine the precise position when the football hit the sensor plate. Thus the continuation of the trajectory can be predicted more accurately. One sensor is placed underneath the football. An array of sensors is attached to the frame that is placed behind the screen to measure the position where the football will hit. The force sensor array consists of 56 FSR-406 force sensors from Interlink Electronics, which are single-zone Force Sensing Resistors (FRS). FRSs are two-wire devices. They are polymer thick film (PTF) sensors that exhibit a decrease in resistance with increase in force applied to the surface of the sensor. A FSR-406 sensor has the dimension of $1.5 \mathrm{in} \times 1.5 \mathrm{in}$ active area. The sensor under the football is used to determine the time instant when the football leaves the ground, the sensor array is used to determine the moment the ball hits the screen as well as the given position and resulting angle for each location. Using these two time stamps between the ground and the sensor array, the average velocity of the football during this interval can be calculated. When multiple sensors are hit, the force sensors will be used in a comparative fashion such that the sensor that reads the highest force value will be registered and used to simulate the rest of the football trajectory.

For proper mounting, these force sensors are covered by 6 in $x$ 6in plexiglass shields to increase the area coverage for each single sensor. The total area of coverage is $4 \mathrm{ft} \times 3.5 \mathrm{ft}$. in a grid of eight by seven sensors. The adhesive backing on the force sensors allows the sensors to be placed semi-permanently to the steel sheet metal. A spherical disk is created with a 0.38 in thick center and tapers off to .28 in thick edge with a 1.6in diameter, attached to the center of the shield. The dimensions and design of spherical disk was designed to optimize contact with the sensor in such a way that the force applied to the shield's corners and/or the sides can be dispersed evenly so that the full impact force from the plexiglass shield can be transferred to the sensor. This design ensures the sensitivity and consistency of the force applied on the shield being relayed back to the force sensor.

\section{A. Data-Driven Quantitative Training}

To use this system for training, the football will be kicked from $8 \mathrm{ft}$ away from the kicking simulator screen, which allows for the football to travel between a $30^{\circ}$ to a $45^{\circ}$ kicking angle in the vertical direction, which is an effective range for an on-field football kick. This also allows for a $10.6^{\circ}$ kicking angle to the left or right of a center kick that is more than the maximum angle allowed in a zero cross-wind kick (i.e. $8.76^{\circ}$ kick) to the left or right of center. The $8.67^{\circ}$ angle may occur during a point after kick known as an extra point kick, which is kicked from approximately 20 yards (60 ft.) away from the field goal post that has an $18.5 \mathrm{ft}$. width. Each force sensor has its own vertical angle $(\theta)$ and horizontal angle from the left or right of center $(\varphi)$, and vertical, horizontal and total distance from where the football is initially kicked. As mentioned previously, there is a force sensor placed under the football to mark the initiation of football trajectory. When the football is kicked, the sudden force reduction provides a time stamp, as soon as the football hits the force sensor array another time stamp is recorded. Based on the elapsed time between the two time stamps and the known distances to the individual sensors, it is possible to calculate the velocity of the ball at the moment it hits the force sensors. The calculated velocity, given distances, 
and each angle $(\theta, \phi)$ provide the initial data for the computer model to predict the remaining portion of the trajectorybased on a dynamic model by considering all six degrees of freedom of the flying football under the influence of drag, lift and wind factors. Unlike other football simulators mainly used for entertainment, this FKS system is designed for training based on real-time football flight data and accurate computer modeling by emulating the realistic environment (see Fig. 2). To achieve the goal of highest conceivable authenticity requires advanced fluid dynamic equations and a great amount of variables received from sensors. The information received from the different sensors can be used as input for the equations in order to create a better prediction of the trajectory to be presented in the visualization. With this consideration, kicker-specific statistics can be logged for each player using the system so they and their coaches can keep track of progress and inspect for consistency. Since the FKS system collects the kicking angle data of the trajectory, football coaches are provided with a better understanding of a player's ability to kick at different hash marks on the field. All the data received while practicing with this simulator can also be used for designing kicker-specific training strategies and drills on the field. In addition, advanced models such as fluid-structure interaction modeling of deformable football can be included, which is out of scope of this paper.

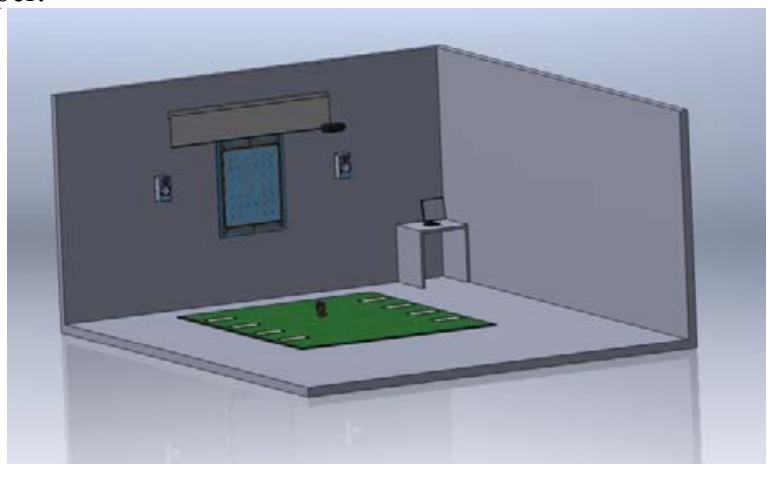

Figure 2 ILLUSTRATION OF FOOTBALL KICKING SimUlator AND TRAINING SYSTEM INSTALLED IN A TRAINING ROOM CONSISTING OF Pressure Plate Mounted on the WALl AND Foot-Print Pressure MAT ON THE FLOOR.

\section{Real-Time SEnsing AND Data Collection}

In order to accurately reconstruct the trajectory of a football, flight data must be captured in real time for model calibration. Moreover, the initial velocity, imparting angles, and rotational velocity of the football must be determined as input for football dynamics analysis. In our design, data are acquired through the combined interaction of several computing devices. Specifically, two microcontrollers will interact with various sensors and send updated information to a computer. An android smartphone implementation will allow users to control the FKS device through a wireless touchscreen interface. Either a laptop or desktop computer will receive the necessary information to reconstruct a 3D simulation. The final visualization with predicted football flight trajectory would be shown on a projection screen that is overlaid on the pressure plate. The central microcontroller (Arduiano/Uno) will receive commands from the smartphone while simultaneously receiving updated data from a wireless sensor (Arduian/Nano). As data are updated from the wireless sensors and force sensors connected to the central microcontroller; variables will be updated in real time and sent across a serial connection to a personal computer. The block diagram in Fig. 3 shows how the control modules interact with the sensors.

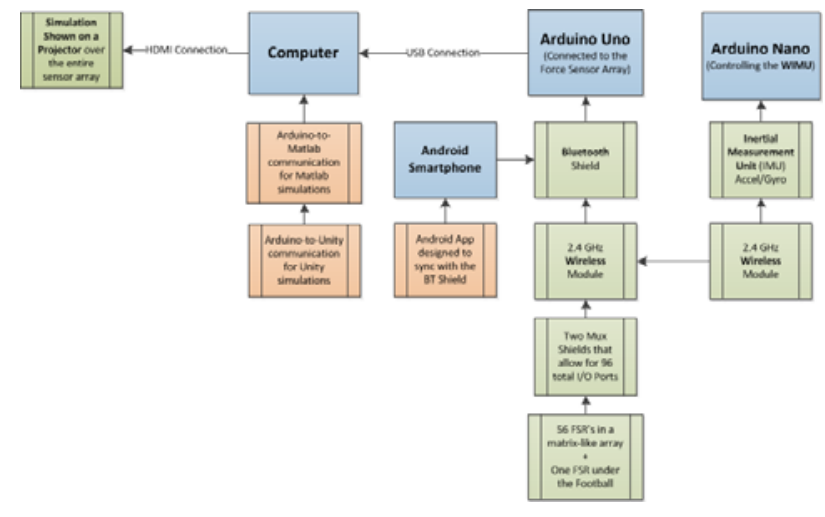

Figure 3 FUnCTIONAL BLOCK DIAGRAM For CONTROL AND DATA COMMUNICATION.

In the current prototype system, an array of 56 Force Sensitive Resistors (FSRs) is connected to an Arduino/Uno to multiplexer shields. All 56 sensors are being scanned at around 200 times per second, and the Arduino is able to determine which one was impacted by keeping track of which sensor has received the most maximum impulse. Once the Ardriano/Uno has received an impact signal, the microcontroller will quickly determine the imparting angles and initial velocity. At this point the Uno will ping the WIMU sensor one last time to get any updated information. Now that the Uno has collected all necessary information, a signal will be sent across the serial connection to a PC with the simulation software. As the simulation software receives a batch of fresh variables, the computer will calculate a trajectory and display such visuals on the projector screen. This entire process happens in fractions of a second. The realtime prediction provides users (football kickers and coaches) with seamless and realistic visualization.

In the following, a brief description of smartphonebased control framework is provided. First, the central microcontroller (Arduiano/Uno) will receive commands from the smartphone while simultaneously receiving updated data from a wireless sensor (Arduiano/Nano) as data is updated from the wireless sensors and force sensors. The pseudo codes of various modules are listed below.

\section{Central Unit Module}


If initial conditions are still 0

Turn on Red light

Scan Force Sensors

Find maximum value across all sensors

Check which distance and angles are associated with the current maximum

Calculate elapsed time

If a starting time has been recorded

Turn on Yellow Light

Scan Force Sensors

Check which distance and angles are associated with the current maximum

Calculate elapsed time

If all necessary data has been recorded

Turn on Green Light

Scan Force Sensors

Check which distance and angles are associated with the current maximum

Calculate elapsed time

Control Module

If the football is placed on the tee

Reset all data variables to zero

If "reset" button is pressed

Reset all data variables to zero

If "stream" button is pressed

Turn stream mode on

If "stream off" is pressed

Turn stream mode off

If "stream mode" is on

Ping the WIMU module at each loop iteration

If "print all" button is pressed

Print all FSR input values to screen

If "print" button is pressed

Print all data variables to screen

If "ping" button is pressed

Ping the WIMU sensor once

If "timer" button is pressed

Set loop counter to ten

If loop timer is greater than zero

Calculate elapsed time and decrement counter

Else

Reset touchscreen input variable

WIMU Module

Inertial Measurement Unit (IMU) Print and Sort $I M U$

Read values for all six pieces of information from the

Compare new data with the current maximums

If new $x$-axis accelerometer value is larger than the current maximum

Replace $x$ accelerometer maximum with current value

If new y-axis accelerometer value is larger than the current maximum

Replace y accelerometer maximum with current value

If new z-axis accelerometer value is larger than the current maximum

Replace $z$ accelerometer maximum with current value

If new $x$-axis gyroscope value is larger than the current maximum
Replace $x$ gyroscope maximum with current value

If new y-axis gyroscope value is larger than the current maximum

Replace y gyroscope maximum with current value

If new z-axis gyroscope value is larger than the current maximum

Replace z gyroscope maximum with current value

Ping Function

If "ping" is received from the central unit

Store ping data to input variable

If input is 1

Reset all variables to zero

If input is 2

Send data in a response to the central unit

\section{BOdy Motion AND TRAJECTORY PREDICTION}

Football dynamics equations are established based upon Newton's Second Law. Lift and drag forces on the football are considered and evaluated as the ball is rotating. The data received from multiple sensors are initial velocities in the $\mathrm{x}, \mathrm{y}$, and $\mathrm{z}$ directions, the rotation speed of the football, and angles of trajectory. A standard right-handed coordinate system is used, where the $\mathrm{x}$ direction is defined as the horizontal distance towards the field goal post, $y$ is the horizontal distance to the left and right from the center where the football was kicked, and the $\mathrm{z}$ is the vertical direction.

Due to the oblong shape of the American football and the way it rotates in the air, the cross sectional area of the ball with respect to drag and lift has a great effect on the trajectory. Drag and lift due to Magnus force are solved by calculating the cross sectional area $\mathrm{S}$ of a prolate ellipsoid as view from the direction of motion [7].

$$
A=\pi a b^{2} \sqrt{\left(\frac{\sin ^{2} \varphi}{a^{2}}+\frac{\cos ^{2} \varphi}{b^{2}}\right) \sin ^{2} \varepsilon+\frac{\cos ^{2} \varepsilon}{b^{2}}}
$$

In the equation above, $\varepsilon$ cis the angle between the viewer and the axis of rotation, $\varphi$ is the angle of rotation. In our case, is the angle between the velocity vectorof $v$ the football and axis of rotationrof the football. In addition, we approximate the long and short radii of the NCAA football as $a=0.113$ $m \mathrm{a}=0.113 \mathrm{mand} b=0.085 \mathrm{~m}$, respectively. These are used as the semi-axes of the prolate spheroid.

The coefficient of drag as a function of the Reynold's number for a sphere is obtained based on [1]. In our case, theReynold's number ranges from $4.4 \times 10^{4}$ and $4.0 \times 10^{5}$ for the football speed between $7 \sim 35.7 \mathrm{~m} / \mathrm{s}$.In this range, Reynold's numbers the drag coefficient is relatively flat. Thus, we assume the drag is independent of Reynold's 
number. Values of drag coefficients given different $\varphi$ are obtained from experiments and simulations done on an AFL ball $[5,6]$. We then use the approximation of the form an analytical function of $\varphi$,

$$
C_{\text {drag }}=0.1+\frac{\text { range } *(1-\cos (2 * \varphi))}{2}
$$

whererange range is a constant that is approximated to be 0.5 . This provides the maximum value of 0.6 for $C_{d r a g} C_{d r a g}$, which agrees with the broadside drag of the American football given by Brancazio [9]. The drag force $D$ Dis then calculated with the basic drag force formula [3][3].

$$
D=C_{\text {drag }} \frac{1}{2} \rho_{\text {air }}\|v\|^{2} A
$$

In addition, the Milne-Thomson equation for lift on a sphere has been used for the calculation of lift force due to the Magnus effect [2]. For a sphere of radius $b$ the equation can be derived from the integration of the Magnus effect for cylinders that compose the sphere. This integration results in the following lift force,

$$
F_{L}=C_{\text {lift }} \frac{4}{3}\left(4 \pi^{2} b^{3} s \rho\|v\|\right)
$$

where $v$ is the velocity of the sphere, $\mathrm{s}$ is revolutions per second of the sphere, $\rho \rho$ is the density of the medium the sphere is moving through. Since the lift due to the Magnus effect is independent of any single angle $\varphi$, the lift coefficient $C_{\text {lift }} \mathrm{C}_{\text {lift }}$ is approximated as a constant of .2, given the experimental data and simulations $[4,8,9]$. Similarly, for a prolate spheroid, the integration can be performed resulting in

$$
L_{\text {Magnus }}=C_{\text {lift }} \frac{4}{3}\left(2 \pi a b^{2} \omega \rho_{\text {air }}\|v\|\right)
$$

where the only change significant change is due to the elongation of the sphere, represented by $a b^{2}$ term. In addition, $\omega=2 \pi s \omega=2 \pi \mathrm{s}$ in the equation.

Now, the position of a football can be determine by the following second order system of differential equations.

$$
\begin{gathered}
\left(L_{x}, L_{y}, L_{z}\right)=L_{\text {Magnus }}\left(\frac{v}{\|v\|} \times r\right) \\
\left(D_{x}, D_{y}, D_{z}\right)=D \frac{v}{\|v\|} \\
m \frac{d^{2} x(t)}{d t^{2}}=\mathrm{L}_{\mathrm{x}}-D_{x}\left\|\frac{d x(t)}{d t}\right\| \frac{\frac{d x(t)}{d t}}{m \frac{d^{2} y(t)}{d t^{2}}=\mathrm{L}_{\mathrm{y}}-D_{y}\left\|\frac{d y(t)}{d t}\right\| \frac{d y(t)}{d t}}
\end{gathered}
$$

$$
m \frac{d^{2} z(t)}{d t^{2}}=\mathrm{L}_{\mathrm{z}}-D_{z}\left\|\frac{d z(t)}{d t}\right\| \frac{d z(t)}{d t}-m g
$$

where $m$ is the mass of the football, $\mathrm{L}_{\text {Magnus }} L_{\text {Magnus }}$ is the lift force due to the Magnus effect, $\mathrm{D} D$ is the drag force, $\omega \omega$ is the angular velocity, $\mathrm{r} r$ is the axis of rotation, Vvis the velocity of the ball, and $g$ is gravity. Using the forth order Runge-Kutta method, or any other numerical methods for that matter, these equations can be solved with explicit values for displacement of the ball. These values are in turn used for visualization of the trajectory in the simulation environment. The model can later be calibrated using real-time data from the WIMU device embedded in the football.

\section{REALISTIC VISUALIZATION FOR TRAINING}

The visualization component is intended to recreate playing conditions. By implementing a digitized Dallas Cowboys stadium, parameterizing the distance and angle of approach, and recreating gameplay sounds the kicker will feel immersed in a true game scenario. A room mock up is shown in figure 11 comprising a graphical user interface and projection system. The visualization algorithm is implemented using Unity [4], an open source interactive gaming and visualization program which allows' 3D components to be imported to construct the environment for the simulator with realistic sceneries. This virtual simulation uses appropriate perspective and scaling to emulate game-time senarios, creating an immersive environment for the kicker.

Information about the screen contact position, in-flight rate of rotation, and velocity just prior to the point of contact are obtained as described above and sent to the Unity Simulation. The in-flight rate of rotation $(\omega)$ and instantaneous velocity (v) at the point of contact are then used to solve the first-order form of the trajectory PDEs. The initial conditions for the PDEs are determined by the instantaneous velocity $\mathrm{v}$, while the rate of rotation $\omega$ is a constant that is used to adjust the lift force. The PDEs are then solved with a JavaScript based 4th order RungeKutta solver. To obtain the final trajectory of the football in flight, a JavaScript based trapezoidal integration algorithm is used.

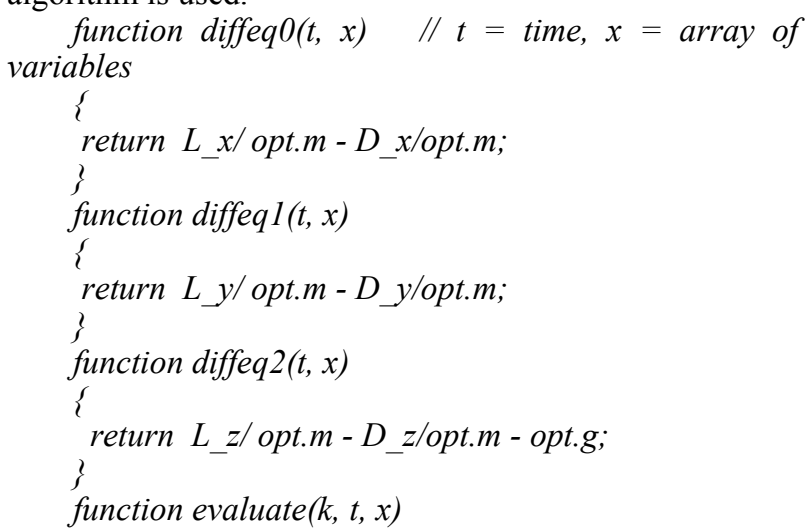


\{

switch $(k)$

\{

case 0: return diffeq $0(t, x)$;

case 1: return diffeq $1(t, x)$,

case 2: return diffeq2 $(t, x)$;

default:

\}

return 0 ;

f

// A version of Runge-Kutta method using arrays

$/ /$ Calculates the values of the variables at time $t+h$

$/ / t=$ last time value

$/ / h=$ time increment

$/ / v=$ array of variables

$/ / N=$ number of variables in $x$ array

function solve $(t, h)$

\{

for $(i=0 ; i<N ; i++)$

$k l[i]=$ evaluate $(i, t, v) ; \quad / /$ evaluate at time $t$

for $(i=0 ; i<N ; i++)$

inp $[i]=v[i]+k 1[i] * h / 2 \quad / /$ set up input to diffeqs

for $(i=0 ; i<N ; i++)$ $t+h / 2$

$k 2[i]=$ evaluate $(i, t+h / 2$, inp $) \quad / /$ evaluate at time

for $(i=0 ; i<N ; i++)$

inp $[i]=v[i]+k 2[i] * h / 2 / /$ set up input to diffeqs

for $(i=0 ; i<N ; i++)$ $t+h / 2$

$k 3[i]=$ evaluate $(i, t+h / 2$,inp $) \quad / /$ evaluate at time

$$
\begin{aligned}
& \text { for }(i=0 ; i<N ; i++) \\
& \quad \text { inp }[i]=v[i]+k 3[i] * h \quad / / \text { set up input to diffeqs } \\
& \quad \text { for }(i=0 ; i<N ; i++) \\
& \quad k 4[i]=\text { evaluate }(i, t+h, i n p) \quad / / \text { evaluate at time }
\end{aligned}
$$$$
t+h
$$$$
\begin{aligned}
& \text { for }(i=0 ; i<N ; i++) \\
& v v[i]=v[i]+(k 1[i]+2 * k 2[i]+2 * k 3[i]+k 4[i]) * h / 6
\end{aligned}
$$$$
\text { \} }
$$$$
\text { function simulate() }
$$$$
\{
$$$$
t=0
$$$$
\text { for }(k=1 ; k<\text { steps; } t=t+h)
$$$$
\{
$$

CalculateCoefficients()

solve $(t, h)$

// Trapazoidal integration

for $(\operatorname{var} j=0 ; j<N ; j++)$

\{

$$
\begin{gathered}
v 2[j][k]=v 2[j][k-1]+h *(v[j]+v 1[j][k-1]) / 2 \\
v 1[j][k]=v[j]
\end{gathered}
$$$$
\text { \} }
$$

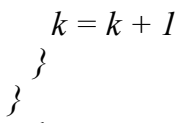

The screen contact position is then used to position the trajectory, and the Unity engine is used to transform the coordinates into world-space and position the virtual football. If the football passes within a predefined region above the virtual goal post crossbeam, and between the two uprights, the kick is considered "good"; otherwise, the kick is considered "no good". A notification is displayed informing the player of the outcome of their kick. An option to display a virtual football trail allows the player tosee the trajectory of the football. Figure 4 shows a screen shot of the simulation environment.

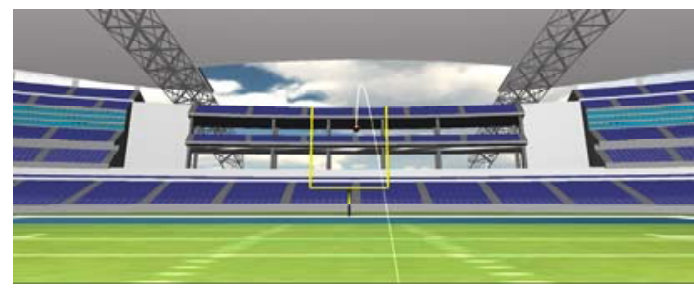

FIGURE 4 VISUALIZATION OF TRAJECTORY

\section{CONCLUSION}

This virtual training system has been developed to include force sensing pads, visualization software, accelerometer, micro-controllers, wirelessinertial measurement unit (WIMU), and Bluetooth. The system works by using force and motion sensing to collect dynamic data and wirelessly transmit it to a nearby computer. Avisualization comprising a realistic football stadium scene and reliable trajectory assures players and coaches are receiving accurate data. The structure design is meant forinside use and can be mounted on a wall or altered for portable use. The few steps left in the prototype include incorporating high-speed cameras, speakers, and a sensory mat for a foot print trail. The vision for this invention is to one day see it implemented in the training of every serious football team in the nation. The use of technology and advanced training methods are what will set apart major teams. This simulation is on the cutting edge of training technology and with minor adjustments to dynamic equations can be fitted for multiple other sports.

\section{REFERENCES}

[1] J. Owen, "The effects of linear and quadratic drag on falling spheres: an undergraduate laboratory." Institute of Physics Publishing European Journal of Physics, pp. 1085-1091, 2005.

[2] W.P. Huang, G. Sheng, and N.G. Guo, "Finite Element Optimization Design on the Back Frame of the Jaw Crusher PC5282" Advanced Materials Research, vol. 472, 2012, pp. 20242028.

[3] F.M. White,. Fluid Mechanics. $4^{\text {th }}$ ed. Boston; Toronto: McGrawHill, 1999

[4] Vance, A. J., J. M. Buick, and J. Livesey. "Aerodynamics of a rugby ball." Transactions of the ASME-E-Journal of Applied Mechanics 79, no. 2 (2012): 021020. 
[5] Alam, Firoz, Aleksandar Subic, Simon Watkins, and Alexander John Smits. "Aerodynamics of an Australian rules foot ball and rugby ball." In Computational Fluid Dynamics for Sport Simulation, pp. 103-127. Springer Berlin Heidelberg, 2009.

[6] Taketo Mizota, Kazuya Seo, Shinichiro Ito, and Hideki Takagi. The Japan Society of Mechanical Engineering. Vol. 15, No.2, December 2004.

[7] M. Festou, H.U. Keller, H.A. Weaver, "Comets II" ; University of Arizona Press, 2004.

[8] M. Verfahrenstechnik, Z. für Ingenieurwissenschaften, M. HalleWittenberg, "Lattice Boltzmann simulations to determine drag, lift and torque acting on non-spherical particles" Computers \& Fluids, pp. 572-589, 2009

[9] P. Brancazio, "The Physics of Kicking a Football" The Physics teacher, pp. 403-407, October 1985

[10] A. Stout, E. Biglar, J. Kantor, C. Meyers, A. Schaefbauer, Y. Feng, "A Virtual Training System Based On Computer Sensing and Football Kicking Dynamics Using Real-Time Wireless Feedback," Proceeding of 2013 Summer Simulation Multi-Conference, Torento (In Press) 